\title{
Hydrogen Permeation in Fusion Materials and the Development of Tritium Permeation Barriers*)
}

\author{
Anne HOUBEN, Marcin RASINSKI and Christian LINSMEIER \\ Forschungszentrum Jülich GmbH, Institut für Energie- und Klimaforschung - Plasmaphysik, Partner of the Trilateral \\ Euregio Cluster (TEC), 52425 Jülich, Germany
}

(Received 29 November 2019 / Accepted 16 February 2020)

\begin{abstract}
Fuel retention and hydrogen permeation in the first wall of future fusion devices are crucial factors. Due to safety issues and in order to guarantee an economical reactor operation, tritium accumulation into reactor walls and permeation through walls have to be estimated and prevented. Therefore, studies of permeation in the fusion materials are performed and the need for tritium permeation barriers (TPB) is verified. The development of TPB layers is explained. A reliable way of comparing different TPB layers and the estimation of the permeation reduction effect of a TPB layer on different bulk materials is enabled by calculation of the layer permeability.
\end{abstract}

(C) 2020 The Japan Society of Plasma Science and Nuclear Fusion Research

Keywords: gas-driven hydrogen permeation measurement, tritium permeation barrier, yttrium oxide, reduced activation ferritic martensitic steel, $316 \mathrm{~L}(\mathrm{~N})-\mathrm{IG}$ steel

DOI: $10.1585 / \mathrm{pfr} .15 .2405016$

\section{Introduction}

Hydrogen permeation through reactor wall materials was identified as a crucial problem for reactor operation already at the beginning of the fusion reactor development. Permeation studies on first-wall materials, especially steels, were performed in the 1970s [1,2]. The aim of these studies was the predictability of the wall loading with hydrogen, since fuel loss and fuel desorption from the wall are critical points for a successful and economical reactor operation. Furthermore, it is an important safety issue that the radioactive tritium is not released into the environment.

There are two major tritium sources in the fusion reactor. The first source is the plasma that is why permeation studies on first wall materials, e.g. steels, graphite, beryllium and tungsten, were performed. The second source is the tritium breeding unit. The steel tubes of the breeding blanket show a high hydrogen permeability.

The need for a tritium permeation barrier (TPB) and the required characteristics of such a barrier material depend on the design and choice of materials used in a fusion device. Furthermore, the required characteristics of a TPB depend on the location in the fusion device: If the application is foreseen in the first wall in order to protect the structural materials from fuel retention, the TPB has to be high temperature, neutron and fast ion resistant. In case of application in the breeding blanket it is also very important, that the TPB materials withstand the lithium or lithium-lead, meaning that the materials have to be corrosion resistant [3]. Therefore, the development and characterization of different TPB materials are required in order

author'se-mail: an.houben@fz-juelich.de

*) This article is based on the presentation at the 28th International Toki Conference on Plasma and Fusion Research (ITC28). to find a suitable material for each specific application.

In order to obtain the hydrogen permeability of a material, there are mainly two types of laboratory experiments: hydrogen-gas-driven permeation or hydrogen-iondriven permeation measurements. These methods are complementary. With the gas-driven permeation measurement, the clean and smooth surface should not be changed during measurement. Therefore, one obtain fundamental information about the permeation through the bulk of the material, without influence of the surface or surface changes by ions or plasma. The ion-driven method is more applicationoriented, since the hydrogen in the fusion reactor impinge the wall as high energetic ions or neutrals. Due to the fact, that the surface will be changed during the measurement, the determination of the permeation parameters is complex. Most measurements are done with the hydrogen isotope deuterium, because tritium is radioactive and has a small availability and with hydrogen the background of the measurement is much higher and complicated to determine. In general, it is assumed that according to the classic rate theory the ratio of diffusivities of hydrogen isotopes is equivalent to the inverse ratio of the square root of the masses of the isotopes [4]. In the following, the focus is on gas-driven-deuterium permeation measurements.

\section{Deuterium Permeation through Single Component Samples and Substrates}

The permeation flux $J_{P}$ through the bulk of the sample is dependent on the solubility and the diffusivity of hydrogen in the material. Furthermore, surface processes, like dissociation of the deuterium molecule, ad- and absorption and desorption, can enhance or reduce the permeation flux. 
In the diffusion-limited case, in which the rate determining process is diffusion and surface processes are quick and therefore negligible regarding limiting the permeation process, the permeation flux can be expressed by

$$
J_{P}=\frac{P_{0} \sqrt{p}}{d} e^{\frac{-E_{P}}{R T}},
$$

wherein $P_{0}$ and $E_{P}$ are the permeability constant and activation energy, respectively, $d$ is the thickness of the sample, $\mathrm{R}$ is the ideal gas constant and $\mathrm{T}$ the temperature. By measuring the permeation flux through the sample pressure and temperature dependent, the permeability constant and the permeability activation energy can be obtained by an Arrhenius plot. Furthermore, from the pressure dependence, the limiting regime can be derived. In the diffusionlimited case, the permeation flux is proportional to the square root of the pressure, see Equation 1. If the surface processes are limiting the permeation flux, the flux is proportional to the pressure. By the slope of the pressure dependence one obtains information about the limiting regime. Details of the measurement setup and the measurement procedure can be found in $[5,6]$.

Permeation measurements on polycrystalline and nondefect-free samples give information about the effective permeation. In opposite to the lattice permeation, which could be measured on a polished, defect-free single crystal, the effective permeation is influenced by permeation through grain boundaries, traps, interfaces and other defects. In general, grain boundaries will increase the permeation flux, whereas traps will first decrease the permeation flux until saturation of the traps with hydrogen [5]. The influence of these effects can be studied by comparison of different samples of the same material and a pre- and post-analysis of the sample state before and after the permeation measurement. The sample state can be analyzed for example by X-ray diffraction (crystal phase), scanning electron microscopy (microstructure) and X-ray photoelectron spectroscopy (composition). An example of a large influence of the microstructure on the effective permeability will be shown below.

A summary of effective hydrogen permeability data through fusion reactor materials is given in the publication of Causey et al. [4]. In this publication, several published data were collected and relationships between the permeability data of the same material classes are given. In Fig. 1 the effective permeability values of this Causey publication, adapted to deuterium, are shown for steels, tungsten, copper and beryllium in solid lines. In dashed lines as comparison, values for $316 \mathrm{~L}(\mathrm{~N})-\mathrm{IG}$ (austenitic steel used in ITER, IG: ITER grade) and Eurofer97 (reduced activation ferritic martensitic (RAFM) steel) measured on our device and published in Houben et al. [6] are shown. By comparing the permeability of these bulk materials, one can conclude that in future fusion devices such as DEMO, in which steels are foreseen as main wall and steels and copper are foreseen as structural materials, the develop-

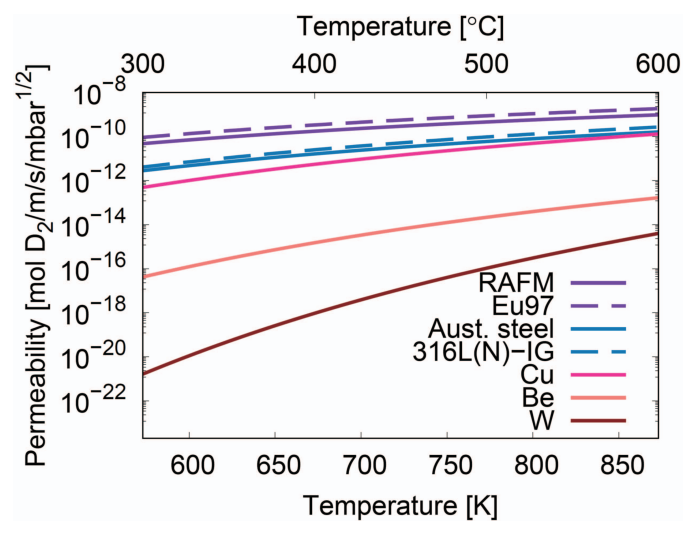

Fig. 1 Deuterium permeability of different fusion materials (bulk) versus temperature: Solid lines: adapted values from Causey et al. [4]: reduced activation ferritic/martensitic steel (RAFM), austenitic steel (Aust. steel), copper $(\mathrm{Cu})$, beryllium $(\mathrm{Be})$ and tungsten $(\mathrm{W})$. Dashed lines: substrate measurements on 316L(N)-IG and Eurofer97 (Eu97) from Houben et al. [6].

ment of TPBs are crucial due to the high permeability of hydrogen through these materials. It was identified that the reduction of the permeation due to the TPB has to be two to three orders of magnitude [7].

\section{Development of a Tritium Permeation Barrier}

Metal oxides were identified as high temperature resistant materials with a low hydrogen permeability already in the $1970 \mathrm{~s}$. Especially $\mathrm{Al}_{2} \mathrm{O}_{3}$ is a promising barrier material for fission and fusion reactor applications. Furthermore, it was noticed that by oxidation of steel a natural oxide layer is produced which reduces the hydrogen permeation. Therefore $\mathrm{Cr}$ and $\mathrm{Fe}$ oxides can also act as permeation barriers. Also nitrides, e.g. TiN, and carbides, e.g. $\mathrm{SiC}$, were investigated regarding the viability as a hydrogen permeation barrier. A detailed overview can be found in Causey et al. [4]. In order to compare different materials the permeation reduction factor (PRF) is used. The PRF is obtained by dividing $J_{P}$ of the non-coated substrate by $J_{P}$ of the oxidized or coated substrate. It has to be noted that the PRF depends on the used substrate, as one can see from the definition. When comparing PRF values of different barriers, on have to keep this in mind. A typical PRF of ceramics for fusion application is in the order of 10 to 1000 .

In the past mainly $\mathrm{Al}_{2} \mathrm{O}_{3}$ was investigated for fusion applications, because it shows a high thermal resistivity, it is commercially available, can be fabricate with various methods and is also used in many other applications. Many research groups investigated the hydrogen permeation reduction of $\mathrm{Al}_{2} \mathrm{O}_{3}$ in the last decades and the studies are still ongoing [8-12]. Between the results of different $\mathrm{Al}_{2} \mathrm{O}_{3}$ samples the permeation reduction factor varied between 
$10^{2}$ and $10^{4}$. This broad variety of PRF values is related to differences in effective permeability of the $\mathrm{A}_{2} \mathrm{O}_{3}$ layers prepared by different methods. This shows, that the microstructure, interfaces, substrate oxidation, crystal phases and many more parameters influence the effective permeation. In order to compare and evaluate a permeation reduction factor, detailed information of the sample and the measurement have to be taken into account.

The requirements on a permeation barrier depends on the application location in a fusion device, as described above. As a rule of thumb, metals show a higher hydrogen permeability than ceramics, due to the reason that metallic bonds exhibit a higher hydrogen permeability than ionic or covalent bonds. As shown in Fig. 1 there are exceptions such as tungsten. It has to be noted that the low permeability through tungsten is due to a very low solubility in the material [4]. The diffusion of hydrogen through tungsten is comparable to other metals and increases drastically at higher temperatures. If one consider a thin tungsten layer as plasma facing material in the first wall, a TPB is still required in order to prevent the structural material (e.g. steel) from a large hydrogen inventory due to a much higher solubility of hydrogen in steels. Next to the requirements for application in a fusion reactor and a high permeation reduction, the possibility of an easy and reliable deposition on a reactor wall part or tubes has to be ensured. The investigated oxides can be deposited via physical vapor deposition, e.g. magnetron sputter deposition, and some can also be deposited via a dip coating process with organic solvent, which is very useful for the layer deposition on tubes. These techniques are widespread in industry and offer a reliable layer deposition.

Regarding the application in the first wall, e.g. between the plasma-facing material (e.g. tungsten) and the structural material, the neutron activation behavior is a crucial parameter to consider. An estimation of the activation of the materials in the wall after a fusion reactor relevant neutron exposure can be found in the 'Handbook of Activation Data Calculated Using EASY-2007' [14] for many elements. Yttrium shows a much shorter activation time after exposure compared to aluminum. Next to the good neutron activation behavior, yttria has many other advantages: only one oxide phase, temperature stable up to around $2000^{\circ} \mathrm{C}$, widespread application in industry, various preparation methods, and commercially available. A detailed overview of the layer deposition preparation and analysis can be found in $[5,13]$. In [13] the influence of the microstructure on the PRF was investigated by measuring the permeation through different $\mathrm{Y}_{2} \mathrm{O}_{3}$ layered substrate. Two kinds of $\mathrm{Y}_{2} \mathrm{O}_{3}$ layers were deposited by reactive magnetron sputtering in different deposition modes, the 'reacted' and the 'hot metallic' mode. The $\mathrm{Y}_{2} \mathrm{O}_{3}$ layers were identical (same crystal structure, composition, thickness and substrate), except the microstructure. Both sides of the substrates were completely covered by the $\mathrm{Y}_{2} \mathrm{O}_{3}$ coating and no cracks or delamination were observed before and

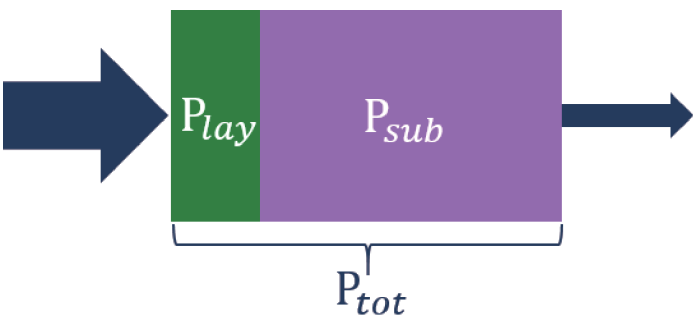

Fig. 2 Illustration of the layer permeability calculation with $P_{t o t}$ : permeability of the layered substrate, $P_{s u b}$ : permeability of the substrate, and $P_{\text {lay }}$ : permeability of the layer.

after permeation measurements by scanning electron microscopy. In the reacted $\mathrm{Y}_{2} \mathrm{O}_{3}$ layer, a porous, equiaxed grain structure was obtained, whereas, the hot metallic $\mathrm{Y}_{2} \mathrm{O}_{3}$ layer was dense at the interface without pores and a columnar structure above the dense part. At $400^{\circ} \mathrm{C}$ and 400 mbar, the PRF of the reacted $\mathrm{Y}_{2} \mathrm{O}_{3}$ was 8 , whereas in the hot metallic $\mathrm{Y}_{2} \mathrm{O}_{3}$ layered sample, the PRF was 600 . The conclusion from this work is that the microstructure largely influences the permeation and by improving the microstructure, the PRF was increased by two orders of magnitude.

\section{Deuterium Permeation through Layered Component Samples}

The deuterium permeation measurement through a substrate which is coated, e.g. by a TPB, is performed the same way as through the bare substrate. Beside the above mentioned disadvantage of the dependence on the permeability of the substrate used, the thickness of the layer is not included in the PRF. In order to express the reduction effect substrate independent and to include the thickness of the layer, the layer permeability is calculated.

The assumption is that the permeability of the layered substrate $\left(P_{t o t}\right)$ depends on the permeability of the bare substrate $\left(P_{\text {sub }}\right)$ and the permeability of the layer $\left(P_{\text {lay }}\right)$, see Fig. 2.

The permeability is regarded as an electrical conductivity and the addition is as resistivity in a series connection. Furthermore, by taking the different thicknesses into account, one obtains the following equation to calculate the layer permeability:

$$
P_{\text {lay }}=\frac{d_{\text {lay }}}{\frac{d_{\text {tot }}}{P_{\text {tot }}}-\frac{d_{\text {sub }}}{P_{\text {sub }}}},
$$

with the thickness of the layer $d_{\text {lay }}$, the thickness of the substrate $d_{s u b}$ and the thickness of the layered substrate $d_{t o t}$. Therefore, by measuring the permeability of the substrate and the layered substrate, the layer permeability can be obtained. As an example, the layer permeability for the $\mathrm{Y}_{2} \mathrm{O}_{3}$ case with two different microstructures discussed above will be estimated. The permeability of the layered substrate can be obtained by an Arrhenius plot and Equation 1 
Table 1 The calculated permeability constant $P_{0}$ and activation energy $E_{P}$ for the bare Eurofer97 (Eu97) substrate [6], the reacted $\mathrm{Y}_{2} \mathrm{O}_{3}$ on Eu97 and hot metallic $\mathrm{Y}_{2} \mathrm{O}_{3}$ on Eu97 samples. These values are mean values of the different applied pressure data and valid in the measured temperature and pressure range only.

\begin{tabular}{l|l|l} 
Sample & $P_{0}\left[\frac{\mathrm{mol}}{\mathrm{ms} \sqrt{\mathrm{mbar}}}\right]$ & $E_{P}\left[\frac{\mathrm{kJ}}{\mathrm{mol}}\right]$ \\
\hline $\mathrm{Eu} 97$ & $5.7(4) \cdot 10^{-7}$ & $41.6(5)$ \\
$\mathrm{Y}_{2} \mathrm{O}_{3}$ reacted on Eu97 & $4(3) \cdot 10^{-10}$ & $12(2)$ \\
$\mathrm{Y}_{2} \mathrm{O}_{3}$ hot metallic on Eu97 & $6(1) \cdot 10^{-9}$ & $53(1)$
\end{tabular}

Table 2 The estimated layer permeability constant $P_{0}$ and activation energy $E_{P}$ for the reacted $\mathrm{Y}_{2} \mathrm{O}_{3}$ and hot metallic $\mathrm{Y}_{2} \mathrm{O}_{3}$ layer, calculated by Equation 2 .

\begin{tabular}{l|l|l} 
Sample & $P_{0}\left[\frac{\mathrm{mol}}{\mathrm{ms} \sqrt{\mathrm{mbar}}}\right]$ & $E_{P}\left[\frac{\mathrm{kJ}}{\mathrm{mol}}\right]$ \\
\hline $\mathrm{Y}_{2} \mathrm{O}_{3}$ reacted & $1 \cdot 10^{-12}$ & 9 \\
$\mathrm{Y}_{2} \mathrm{O}_{3}$ hot metallic & $5 \cdot 10^{-12}$ & 53
\end{tabular}

for both $\mathrm{Y}_{2} \mathrm{O}_{3}$ samples from the data given in $[5,13]$. The calculated permeability is valid in the measured temperature and applied deuterium pressure range only. Details of the permeability calculation and the values for the bare Eurofer97 substrate can be found in [6]. For all samples, the Arrhenius equation is fulfilled in the measured temperature range $\left(300^{\circ} \mathrm{C}\right.$ to $\left.550^{\circ} \mathrm{C}\right)$. The mean values of the activation energy and permeability constant obtained for different applied pressure values ( 25 mbar to $800 \mathrm{mbar}$ ) are given in Table 1. In the substrate and the hot metallic $\mathrm{Y}_{2} \mathrm{O}_{3}$ sample the limiting process is diffusion and the permeation flux is dependent on the square root of the applied deuterium pressure. In the reacted $\mathrm{Y}_{2} \mathrm{O}_{3}$ sample, the rate determining process is in the intermediate regime, meaning that processes on the surface or interface are in the same order as the diffusion process and the permeation flux is not dependent on the square root of the applied pressure. For comparison, a square root dependence was assumed also for the reacted $\mathrm{Y}_{2} \mathrm{O}_{3}$ sample, a mean value was calculated for the activation energy and permeability constant and the deviation from the square root dependence of the applied pressure was considered in the error bars in Table 1 .

From these values given in Table 1 and the thicknesses of the layers, the substrates and the layered substrates, the layer permeability can be estimated with Equation 2. The values for the layer permeability are given in Table 2 and the results are plotted in Fig. 3 in the measured temperature range.

The layer permeability is now independent of the permeation through the substrate and the thickness of the TPB layer is included. Therefore, the estimation of the reduction effect of the hydrogen permeation due to the TPB to other bare substrates is possible, e.g. by comparison to the bulk material permeability shown in Fig. 1. Also, the

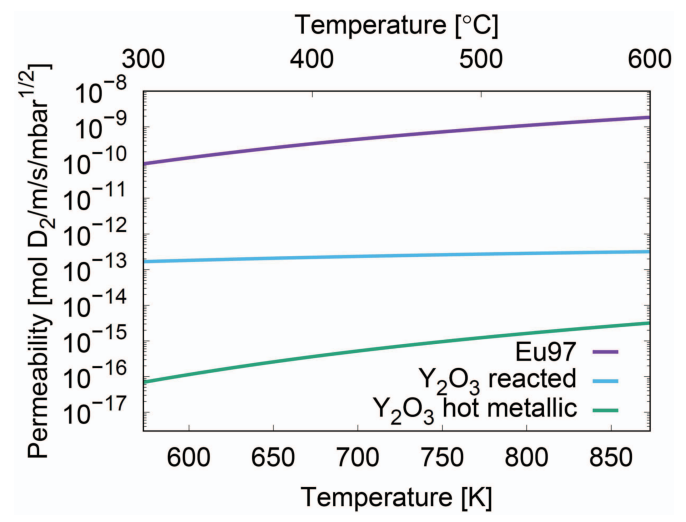

Fig. 3 The calculated layer permeability of the reacted $\mathrm{Y}_{2} \mathrm{O}_{3}$ and the hot metallic $\mathrm{Y}_{2} \mathrm{O}_{3}$. The corresponding parameters can be found in Table 2 and the permeability of the bare Eurofer97 substrate (Eu97) is given as comparison in the figure.

comparison between different TPB layers is more reliable with these parameters as with the comparison of PRF values. Furthermore, the temperature dependence of the permeating flux can be expressed directly from the given parameters for the layer permeability: since the permeability activation energy in the reacted $\mathrm{Y}_{2} \mathrm{O}_{3}$ is smaller than the permeability activation energy in the hot metallic $\mathrm{Y}_{2} \mathrm{O}_{3}$, the temperature dependence is very different in this temperature range, see Fig. 3.

One has to note that the effect of the interface is included in the layer permeability and in the reacted $\mathrm{Y}_{2} \mathrm{O}_{3}$ sample the surface processes as well. In order to study these effects on the permeability, identical fabricated TPB layers with different thicknesses will be studied in the future. With the assumption that the permeation flux through the bulk is the same in all identical fabricated layers and dependent on the thickness of the layer, the influence of the interface and surface can be estimated by this method.

\section{Conclusions and Outlook}

In future fusion devices, TPB layers are crucial in order to guarantee a safe and economical reactor operation. In order to find a suitable TPB layer for every material and location in a fusion device, the development of different kinds of TPB layers which can be adapted to specific applications is necessary. The microstructure of the TPB layer strongly influences the reduction effect, for that reason preand post-analysis of the sample are important. By calculating the layer permeability, a comparison to other TPB layers is more reliable as comparing the PRF value only and the estimation of the reduction effect to other bulk materials and substrates is also enabled by this method. The influence of the interface on the permeation will be studied in the future by varying the layer thickness of a TPB. 


\section{Acknowledgements}

A part of this work has been carried out within the framework of the EUROfusion Consortium (WP PFC) and has received funding from the Euratom research and training program 2014 - 2018 and 2019 - 2020 under grant agreement No 633053. The views and options expressed herein do not necessarily reflect those of the European Commission. The authors would like to thank Dr. Jan Engels for fabrication and characterization of the $\mathrm{Y}_{2} \mathrm{O}_{3}$ samples and the performance of the deuterium permeation measurements.

\section{(c) EURATOM}

[1] F. Waelbroeck, I. Ali-Khan, K. Dietz and P. Wienhold, J. Nucl. Mater. 85, 345 (1979).

[2] I. Ali-Khan, K. Dietz, F. Waelbroeck and P. Wienhold, J. Nucl. Mater. 76, 337 (1978).

[3] Ch. Linsmeier, M. Rieth, J. Aktaa, T. Chikada, A. Hoffmann, J. Hoffmann, A. Houben, H. Kurishita, X. Jin, M. Li, A. Litnovsky, S. Matsuo, A. von Mller, V. Nikolic, T. Palacios, R. Pippan, D. Qu, J. Reiser, J. Riesch, T. Shikama, R. Stieglitz, T. Weber, S. Wurster, J.-H. You and Z. Zhou, Nucl. Fusion 57, 092007 (2017).
[4] R. Causey, R. Karnesky and C.S. Marchi, in Comprehensive Nuclear Materials, edited by R.J. Konings (Elsevier, Oxford, 2012) pp.511-549.

[5] J. Engels, A. Houben, M. Rasinski and Ch. Linsmeier, Fusion Eng. Des. 124, 1140 (2017).

[6] A. Houben, J. Engels, M. Rasinski and Ch. Linsmeier, Nucl. Mater. Energy 19, 55 (2019).

[7] D. Demange, L. Boccaccini, F. Franza, A. Santucci, S. Tosti and R.Wagner, Fusion Eng. Des. 89, 1219 (2014).

[8] K. Forcey, D. Ross and C. Wu, J. Nucl. Mater. 182, 36 (1991).

[9] A. Perujo and K. Forcey, Fusion Eng. Des. 28, 252 (1995), proceedings of the Third International Symposium on Fusion Nuclear Technology.

[10] G. Hollenberg, E. Simonen, G. Kalinin and A. Terlain, Fusion Eng. Des. 28, 190 (1995), proceedings of the Third International Symposium on Fusion Nuclear Technology.

[11] E. Serra, H. Glasbrenner and A. Perujo, Fusion Eng. Des. 41, 149 (1998).

[12] D. Levchuk, F. Koch, H. Maier and H. Bolt, Physica Scripta 2004, 119 (2004).

[13] J. Engels, A. Houben, P. Hansen, M. Rasinski and Ch. Linsmeier, Int. J. Hydrogen Energy 43, 22976 (2018).

[14] R.A. Forrest, A. Tabasso, C Danani, S Jakhar and A.K. Shaw, UKAEA FUS 552 (2009): http://www.ccfe. ac.uk/assets/documents/ukaea-fus-552_0.pdf. 